\title{
Development of Drug-Induced Cutaneous Vasculitis in a Patient with Relapsed Angioimmunoblastic T-cell Lymphoma Treated with Novel Bortezomib and Panobinostat Combination: A Possible Surrogate Marker of Response
}

\author{
Yuh Shan Lee ${ }^{1}$, Leonard Tan ${ }^{2}$, Choon Chiat $\mathrm{Oh}^{3}$, Yuen Li Ng${ }^{4}$, Yeow Tee Goh ${ }^{1}$ and Daryl Tan ${ }^{1 *}$ \\ ${ }^{1}$ Department of Hematology, Singapore General Hospital, Singapore \\ ${ }^{2}$ Department of Pathology, Singapore General Hospital, Singapore \\ ${ }^{3}$ Department of Dermatology, Singapore General Hospital, Singapore \\ ${ }^{4}$ Department of Radiology, Singapore General Hospital, Singapore
}

Received: April 08, 2014; Accepted: June 09, 2014; Published: June15, 2014

*Corresponding author: Daryl Tan, Department of Hematology, Academia, level 3 Singapore General Hospital, College Road, Singapore 169856, Republic of Singapore, Fax : 65-62250210 ; E-mail :daryl.tan@sgh.com.sg, daryltcl1@gmail.com

\section{Introduction}

Relapsed/refractory angioimmunoblastic T-cell lymphoma (AITL) after conventional chemotherapy carries an extremely poor prognosis, and there is currently no recognized effective treatment available. There is hence, an unmet need for novel agents in salvaging patients with relapsed/refractory AITL. Bortezomib exerts pleotropic anti-tumor effects, including inhibition of the NF-KB pathway, leading to cell apoptosis and it has shown promising activity in a variety of lymphoid malignancies [1-4 ]. Panobinostat, a pan-histone deacetylase (HDAC) inhibitor which targets epigenetic and multiple oncogenic pathways has demonstrated potent anti-tumour activity in a variety of malignancies, including T-cell lymphoma [5-6]. In vitro and in vivo studies have demonstrated potent synergistic cytotoxicity of the combination of bortezomib with panobinostat Inhibition of HDAC6 by panobinostat abrogates bortezomibinduced protective aggreosome formation and accentuates bortezomib induced endoplasmic reticulum stress, leading to further apoptosis [7-9]. Collectively, these data provide a strong rationale for the first clinical trial of this novel combination in T-cell lymphoma.

The development of a vasculitic rash has been reported with the use of bortezomib and this has been associated to portend a favorable response to the drug among patients with B-cell nonHodgkin lymphoma treated in clinical trials [10]. Among most cases reported, a perivascular leukocytoclastic vasculitis with no evidence of a systemic vasculitis or hypersensitivity reaction was reported. Herein, we report on a patient with relapsed AITL who responded favourably to the above described combination and developing a typical bortezomib-induced rash after 2 cycles of treatment.

\section{Case Report}

A 50 year old male presented with fever, tonsillar enlargement and cervical lymphadenopathy of 1 month duration. A lymph node biopsy done revealed the presence of a neoplastic T-lymphocytic infiltrate which stained positively for CD3, CD2 and CD7, but weakly for CD5. The association with proliferation of high endothelial venules, eosinophilia and CD21 marking the expanded but disrupted follicular dendritic cell meshwork confirmed the diagnosis AITL. A staging positron emission tomography (PET) scan and bone marrow trephine biopsy demonstrated extensive FDG avid lymphadenopathy above and below the diaphragm, and the absence of marrow involvement by lymphoma respectively. He was commenced on 21-day CHOP (cyclophosphamide, adriamycin, vincristine, prednisolone) chemotherapy for treatment of the stage IIIB AITL. He attained a PET complete response after 4 cycles of CHOP chemotherapy. The response was consolidated with 2 cycles and ICE (ifosfamide, cyclophosphamide and etoposide) regimen, followed by high dose therapy (HDT) with BEAM (carmustine, etoposide, cytarabine and melphalan) conditioning and autologous stem cell support. His response was however, short-lived and 6 months post-HDT, he had a biopsy-proven relapse with involvement of supra and infradiaphramatic lymph nodes, including the cervical and inguinal regions (Figures 1a and 2a). This was also accompanied by bone marrow disease and peripheral blood hypereosinophilia. He was subsequently enrolled into clinical trial NCT00901147 which explores the combination of bortezomib $\left(1.3 \mathrm{mg} / \mathrm{m}^{2}\right.$ on days 1 , $4,8,11$ ) and panobinostat $20 \mathrm{mg}$ ( 3 times per week for 2 weeks) given in a 21-day cycle in relapsed/refractory T-cell lymphoma. Upon completion of the second cycle of the study combination, he developed scattered erythematous papular rash localised to the neck, upper trunk and forearms which were mildly pruritic (Figure 3).He did not have fever, prurisy and autoimmune manifestation. A skin biopsy performed on the lesion over his right forearm demonstrated perivascular lymphoid infiltrates with dyskeratosis of overlying epidermal keratinocytes, consistent 


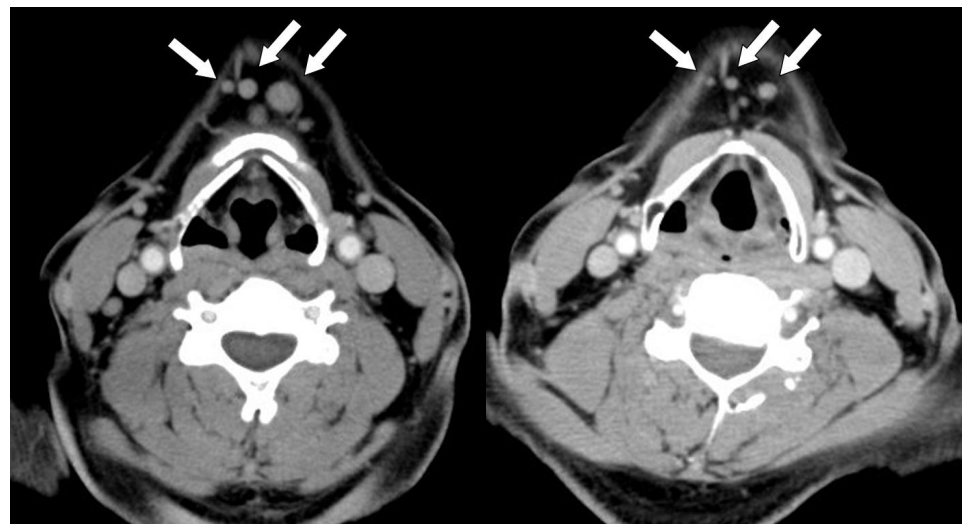

Figure 1: Post therapy.

* White arrows show submental lymph nodes at baseline and after completion of treatment.

Figures 1a and 1b: Axial CT neck; (a) shows submental lymphadenopathy which significantly improved on the post therapy CT (b).
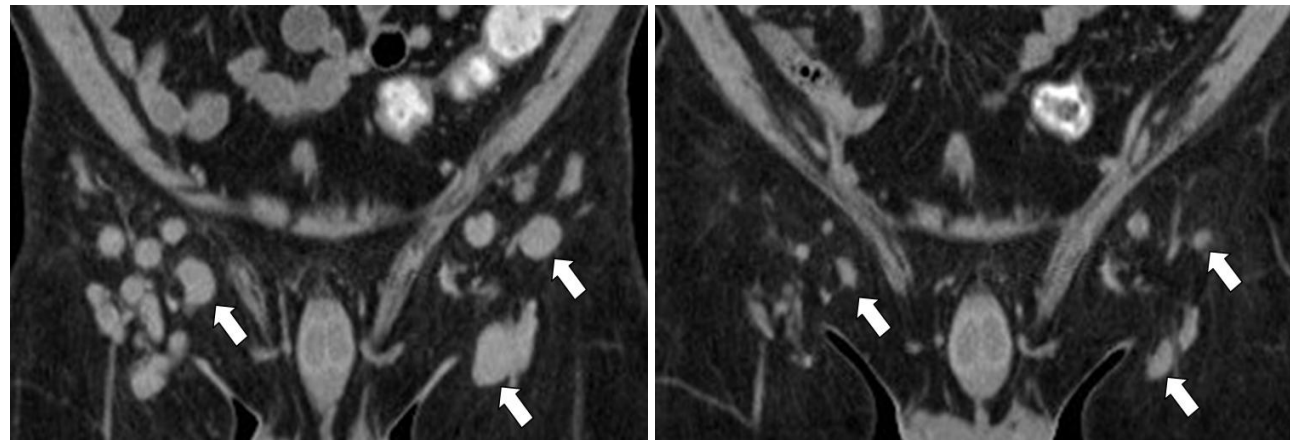

Figure 2: Coronal CT pelvis; (a) shows bilateral inguinal lymphadenopathy (the arrows indicating the 3 largest nodes), which significantly improved on the post-therapy CT (b).

* White arrows show the inguinal lymph nodes at baseline and after completion of treatment.
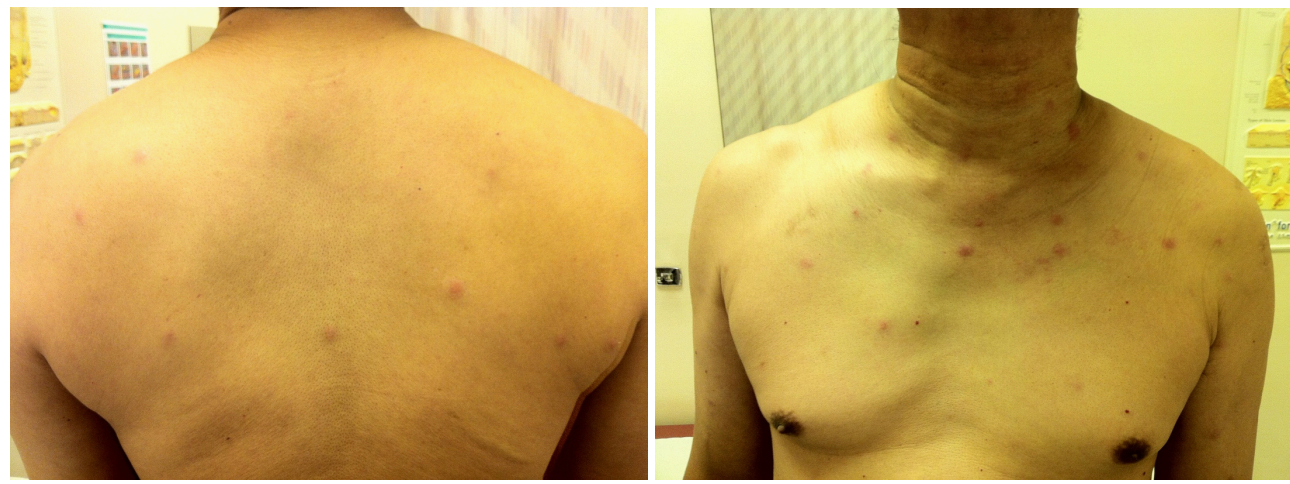

Figure 3: Skin lesions of patient with bortezomib induced rash. Skin lesions of patient with bortezomib induced rash.

with a non-necrotizing cutaneous vasculitis (Figure 4). There was no evidence of lymphoma infiltration or hypersensitivity reaction. The peripheral hypereosinophilia resolved upon commencement of the study drugs. An interim computed tomography (CT) scan done at this juncture demonstrated a partial response by Cheson criteria, with significant interval reduction in size of the lymphadenopathy (Figures $1 \mathrm{~b}, 2 \mathrm{~b}$ ). The rash was self- limiting and resolved spontaneously. A milder eruption of a similar rash occurred with the subsequent administration of the study drugs. The patient continues to respond further.

\section{Discussion}

The causal association of the rash with bortezomib is apparent by the pattern of resolving and recurring rash following cessation and re-introduction of the drug. Development of a rash has not been commonly described as an adverse event with the use of 


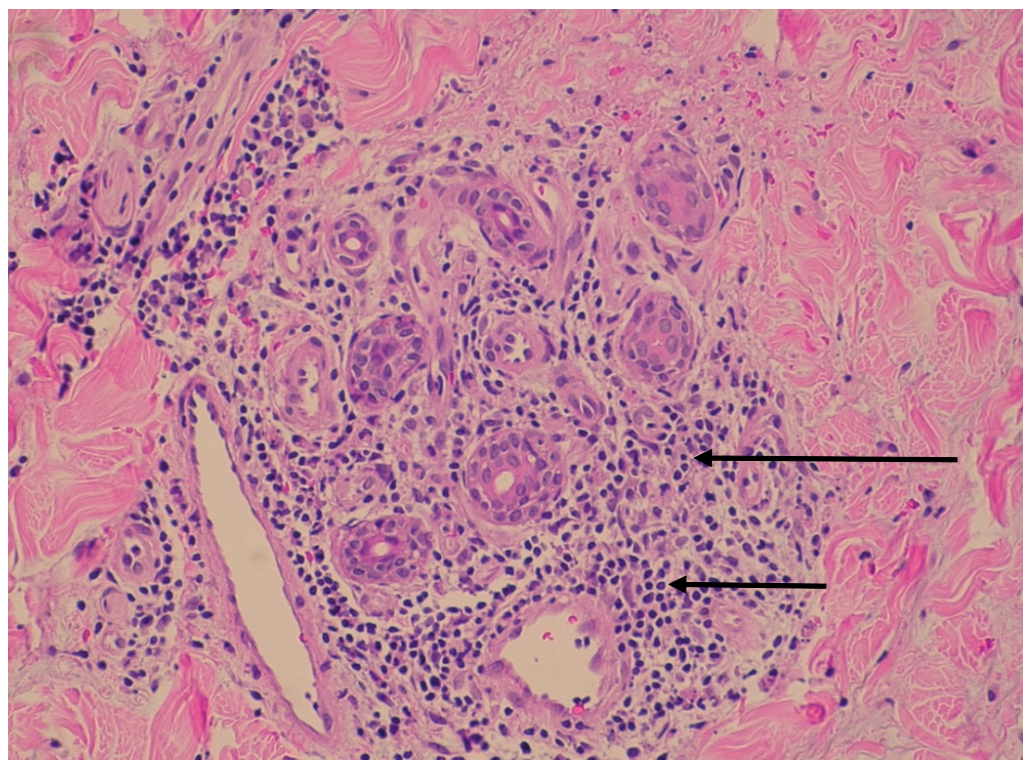

Figure 4: Skin biopsy of patient from right forearm.

* Black arrows showing perivascular lymphoid infiltrates in skin biopsy

panobinostat [6]. There was no evidence of a hypersensitivity reaction and on the contrary, the commencement of the novel combination suppressed the peripheral blood hypereosinophilia, a surrogate of disease activity in AITL. In addition, the skin biopsy excluded the possibility of lymphomatous involvement of the skin, a common occurrence with AITL. To the best of our knowledge, this is the first reported case of bortzomib-induced rash occurring in T-cell non-Hodgkin lymphoma. The first reports of bortezomib-induced rash were among bortezomibtreated patients with multiple myeloma, with reported incidence in clinical trials ranging from 8 to 18\% [11-13]. In myeloma, the correlation between rash and response to treatment could not be further ascertained because glucocorticoids, an integral component of myeloma therapy could dampen the immune response in patients. Although the exact pathogenesis of the rash is unknown, it is likely to be immune-mediated as cutaneous vasculitis is seen in autoimmune condition such as ulcerative colitis. Kim et al. [14] suggest that the pathogenesis of the rash may be related to the overproduction of pro-inflammatory cytokines such as IL-6 and TNF-a. Similar cytokines are also proposed to be the pathogenesis behind bortezomib induced lung injury [15]. Maruyama et al. [16] suggest that the origin of cytokines in bortezomib-treated patient is from bone marrow stromal cells.

Based on results of 3 phase II studies on bortezomib in patients with B-cell non-Hodgkin lymphoma, Gerecitano and colleagues were able to demonstrate a statistically significant positive predictability of response to bortezomib among patients developing the rash, prompting the current recommendation that therapy with bortezomib should not be discontinued in such patients, but rather, continued treatment through the rash with symptomatic relief is to be encouraged [10].

Despite AITL being associated with a dismal median overall survival of less than 3 years, the optimal therapeutic approach for AITL is still unknown as various strategies including intensive chemotherapeutic combinations and stem cell transplantation have not significantly improved survival outcomes. Clearly, novel approaches exploring synergistic combinations are needed. Published work now suggests that targeting both proteasomedependent pathways with bortezomib and the aggresome pathway in tumour cells with HDAC inhibitors induces greater accumulation of polyubiquitinated proteins resulting in increased cell stress and apoptosis [7-9]. Both bortezomib and panobinostat have modest single-agent activity in T-cell lymphoma. The clinical combination of both drugs will help determine if the abundant preclinical data of proteasome inhibitor/HDAC inhibitor synergism could translate into improved clinical outcomes for patients with T-cell lymphoma. This novel treatment combination for AITL is promising and results of our trial in the future will hopefully fill the emergent need for improved treatment strategies in these patients.

\section{References}

1. O'Connor OA, Wright J, Moskowitz C, Muzzy J, MacGregor-Cortelli B, Stubblefield M, et al. Phase II clinical experience with the novel proteasome inhibitor bortezomib in patients with indolent nonHodgkin's lymphoma and mantle cell lymphoma. J Clin Oncol. 2005; 23(4):676-684.

2. Cavo M, Tacchetti P, Patriarca F, Petrucci MT, Pantani L, Galli M, et al. Bortezomib with thalidomide plus dexamethasone compared with thalidomide plus dexamethasone as induction therapy before, and consolidation therapy after, double autologous stem-cell transplantation in newly diagnosed multiple myeloma: a randomised phase 3 study. Lancet. 2010; 376(9758):2075-2085. doi: 10.1016/ S0140-6736(10)61424-9.

3. Harousseau JL, Attal M, Avet-Loiseau H, Marit G, Caillot D, Mohty M, et al. Bortezomib plus dexamethasone is superior to vincristine plus doxorubicin plus dexamethasone as induction treatment prior to 
autologous stem-cell transplantation in newly diagnosed multiple myeloma: results of the IFM 2005-01 phase III trial. J Clin Oncol. 2010; 28(30):4621-4629. doi: 10.1200/JC0.2009.27.9158.

4. San Miguel JF, Schlag R, Khuageva NK, Meletios A. Dimopoulos, Ofe Shpilberg, Martin Kropff, et al. Bortezomib plus melphalan and prednisone for initial treatment of multiple myeloma. N Engl J Med. 2008; 359:906-917. doi: 10.1056/NEJMoa0801479.

5. Ellis L, Pan Y, Smyth GK, George DJ, McCormack C, Williams-Truax $\mathrm{R}$, et al. Histone deacetylase inhibitor panobinostat induces clinical responses with associated alterations in gene expression profiles in cutaneous T-cell lymphoma. ClinCancer Res. 2008; 14(14):45004510. doi: 10.1158/1078-0432.

6. Ottmann OG, Spencer A, Prince HM, H. Miles Prince, Bhalla K.N, Fischer T, et al. Phase IA/II study of oral panobinostat (LBH589), a Novel PanDeacetylase Inhibitor (DACi) Demonstrating Efficacy in Patients with Advanced Hematologic Malignancies. Blood. 2008; 112: Abstract 958.

7. Hideshima T, Bradner JE, Wong J, Chauhan D, Richardson P, Schreiber SL, et al. Small-molecule inhibition of proteasome and aggresome function induces synergistic antitumor activity in multiple myeloma. Proc Natl Acad Sci. USA. 2005; 102:8567-8572.

8. Catley L, Weisberg E, Kiziltepe T, Tai YT, Hideshima T, Neri P, et al. Aggresome induction by proteasome inhibitor bortezomib and alphatubulin hyperacetylation by tubulin deacetylase (TDAC) inhibitor LBH589 are synergistic in myeloma cells. Blood. 2006; 108(10):34413449. doi: 10.1182/blood-2006-04-016055.

9. Bhalla S, David K, Mauro L, Prachand S, Sirisawad M, Balasubramanian $\mathrm{S}$, et al. Histone Deacetylase Inhibitor (HDACi) PCI-24781 and Bortezomib Result in Synergistic Apoptosis in Hodgkin Lymphoma (HL) and Non-Hodgkin's Lymphoma (NHL) Cell Lines: Investigation of Cell Death and NFKB-Mediated Pathways. Blood. 2007; 110: Abstract 3589.
10.J Gerecitano, A Goy, J Wright, MacGregor-Cortelli B, Neylon E, Gonen $\mathrm{M}$, et al. Drug-induced cutaneous vasculitis in patients with nonHodgkin lymphoma treated with the novel proteasome inhibitor bortezomib: a possible surrogate marker of response? Br J Haematol. 2006; 134(4):391-398.

11. Richardson PG, Sonneveld P, Schuster MW, Irwin D, Stadtmauer EA, Facon T, et al. Assessment of Proteasome Inhibition for Extending Remissions (APEX) Investigators. Bortezomib or high-dose dexamethasone for relapsed multiple myeloma. N Engl J Med. 2005; 352(24):2487-2498.

12. Wu KL, van Wieringen W, Vellenga E, Zweegman S, Lokhorst HM, Sonneveld P. Analysis of the efficacy and toxicity of bortezomib for treatment of relapsed or refractory multiple myeloma in community practice. Haematologica. 2005; $90(7): 996-997$.

13. Richardson PG, Barlogie B, Berenson J, Singhal S, Jagannath S, Irwin D, et al. A phase 2 study of bortezomib in relapsed, refractory myeloma. N Engl J Med. 2003; 348(26):2609-2617.

14. Min C-K, Lee S, Kim Y-J, Eom KS, Lee JW, Min WS, et al. Cutaneous leucoclastic vasculitis (LV) following bortezomib therapy in a myeloma patient; association with pro-inflammatory cytokines. Eur J Haematol. 2006;76(3):265-268.

15. Kazutake Y, Harumi Y.M, Michiko M, Makiko Miyao,Yoshimasa Ogawa, Kazuma Ohyashik, et al. Bortezomib therapy-related lung disease in Japanese patients with multiple myeloma: Incidence, mortality and clinical characterization. Cancer Science. 2014; 105(2):195-201. doi: $10.1111 /$ cas.12335.

16. Maruyama D, Watanabe T, Heike Y, Nagase K, Takahashi N, Yamasaki $\mathrm{S}$, et al. Stromal cells in bone marrow play important roles in proinflammatory cytokine secretion causing fever following bortezomib administration in patients with multiple myeloma. Int J Hematol. 2008; 88(4):396-402. doi: 10.1007/s12185-008-0194-0. 